\title{
Adiponectin and thiazolidinedione targets CRTC2 to regulate hepatic gluconeogenesis
}

\author{
Young-Sil Yoon, Dongryeol Ryu, Min-Woo Lee, \\ Sungpyo Hong and Seung-Hoi Koo ${ }^{1}$ \\ Department of Molecular Cell Biology \\ Sungkyunkwan University School of Medicine \\ Suwon 440-746, Korea \\ ${ }^{1}$ Corresponding author: Tel, 82-31-299-6122; \\ Fax, 82-31-299-6239; E-mail, shkoo@med.skku.ac.kr \\ DOI 10.3858/emm.2009.41.8.063
}

Accepted 25 March 2009

Abbreviations: AMPK, AMP activated protein kinase; CRTC2, CREB regulated transcriptional coactivator 2; G6Pase, glucose 6 phosphatase catalytic subunit; PEPCK, phosphoenol pyruvate carboxykinase; TZD, thiazolidinedione

\begin{abstract}
During fasting periods, hepatic glucose production is enhanced by glucagon to provide fuels for other organs. This process is mediated via cAMP-dependent induction of the CREB regulated transcriptional coactivator (CRTC) 2, a critical transcriptional activator for hepatic gluconeogenesis. We have previously shown that CRTC2 activity is regulated by AMP activated protein kinase (AMPK) family members. Here we show that adiponectin and thiazolidinedione directly regulate AMPK to modulate CRTC2 activity in hepatocytes. Adiponectin or thiazolidinedione lowered glucose production from primary hepatocytes. Treatment of both reagents reduced gluconeogenic gene expression as well as CAMP-mediated induction of CRE reporter, suggesting that these reagents directly affect CREB/CRTC2- dependent transcription. Furthermore, adiponectin or thiazolidinedione mediated repression of CRE activity is largely blunted by co-expression of phosphorylation defective mutant CRTC2, underscoring the importance of serine 171 residue of this factor. Taken together, we propose that adiponectin and thiazolidinedione promote the modulation of AMPK-dependent CRTC2 activity to influence hepatic gluconeogenesis.
\end{abstract}

Keywords: adiponectin; AMP-activated protein kinases; CRTC2 protein, human; gluconeogenesis; liver; thiazolidinediones

\section{Introduction}

Glucose homeostasis is tightly regulated in mammals via hormonal or nutritional signals that govern the cellular events in various metabolic tissues. In case of liver, hepatic glucose production is a focal point of regulation, and is repressed during feeding conditions and re-activated upon fasting to meet the fuel requirements for various tissues including brain. The process of de novo glucose production or gluconeogenesis is regulated in part via transcriptional mechanism, and the major enzyme genes such as phosphoenol pyruvate carboxykinase $(P E P C K)$ or glucose 6 phosphatase catalytic subunit (G6Pase) are targets for hormone- and nutrition-dependent modulation of their chronic expression (Chrivia et al., 1993; Arias et al., 1994; Hanson and Reshef, 1997; Hall and Granner, 1999; Herzig et al., 2001). CREB regulated transcriptional coactivator (CRTC) 2, also known as TORC2, is shown to be a major regulator for the transcriptional activation of such genes, and is mainly regulated via phosphorylation-dependent cellular localization by AMP activated protein kinase (AMPK) family of Ser/Thr kinases (Koo et al., 2005).

Regulation of hepatic glucose production is one of the critical means to regulate blood glucose levels in the individuals with type II diabetes. Metformin and thiazolidinedione (TZD) types of antidiabetic drugs have been most widely used to treat type II diabetes, and both of them are shown to regulate hepatic AMPK activity either directly or indirectly (Zhou et al., 2001, LeBrasseur et al., 2006). In case of TZD, it was shown that the reagent enhances production of adiponectin via activation of peroxisome proliferator activated receptor (PPAR) $\gamma$ from adipose tissues, which is linked to the activation of AMPK in various tissues including liver (Nawrocki et al., 2006). However, recent studies displayed that TZD can reduce blood glucose levels even in mice lacking fat cell specific expression of PPAR $\gamma$, suggesting the possibility that this drug may act upon another mechanism in other tissues (He et al., 2003).

Here we report that adiponectin and TZD can act directly upon hepatocytes, and regulate hepatic AMPK activity and CRTC2-dependent transcriptional cascades to modulate glucose metabolism in liver. These data provide clear molecular mecha- 
nism by which endogenous or pharmacological signals modulate hepatic gene transcription to control glucose production from liver.

\section{Results and Discussion}

\section{Adiponectin and rosiglitazone inhibit hepatic gluco- neogenic gene expression}

AMPK was shown to regulate hepatic gluconeogenic gene expression (Foretz et al., 2005). Indeed, infection of adenovirus expressing constitutively active AMPK (AMPK CA) greatly reduces mRNA levels for $P G C-1 \alpha$ and G6Pase in primary hepatocytes (Figure 1A). In addition, adenovirus-mediated expression of adiponectin, an adipokine known to induce AMPK activity in liver, also inhibits gluconeogenic gene expression as well as CRE luciferase activity in primary hepatocytes (Figure $1 \mathrm{~A}$ and $1 \mathrm{~B}$ ), suggesting adiponectin is directly responsible for the regulation of CREB-dependent transcription. Previously, rosiglitazone was shown to be involved in the up-regulation of adiponectin in fat cells, thus regulating hepatic gluconeogenesis in an indirect manner (Nawrocki et al., 2006). Surprisingly, short term treatment of rosiglitazone effectively reduces expression of endogenous gluconeogenic genes as well as CRE luciferase activity (Figure 1C and 1D), suggesting that this reagent could directly act upon hepatocytes to modulate glucose metabolism at the transcriptional level.
A

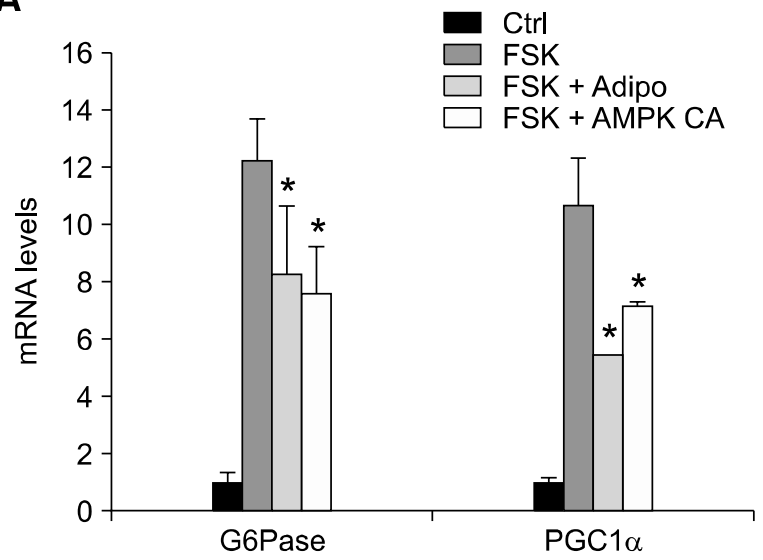

C

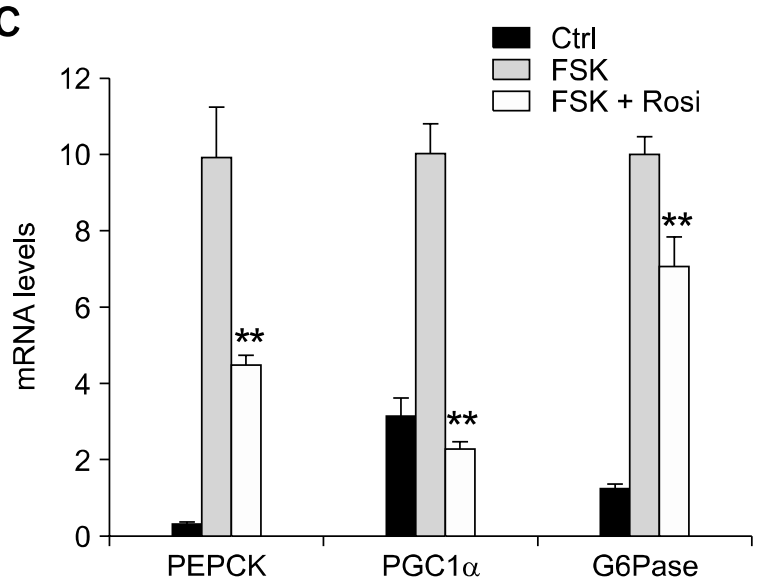

B

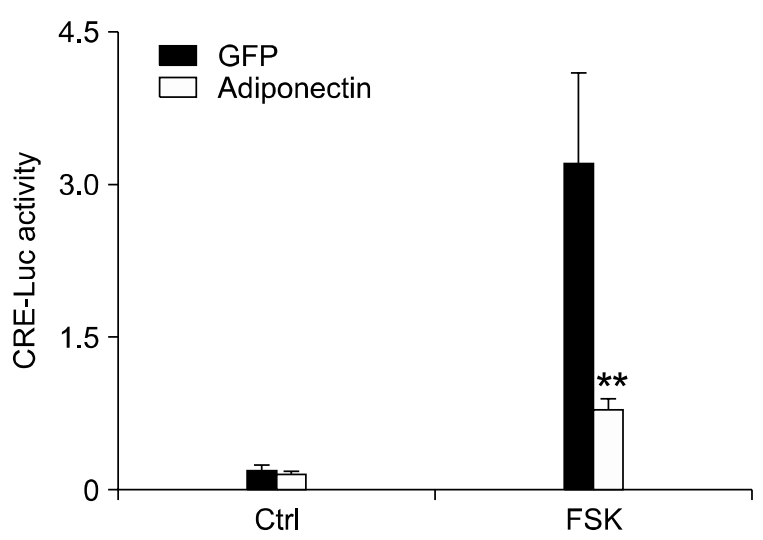

D

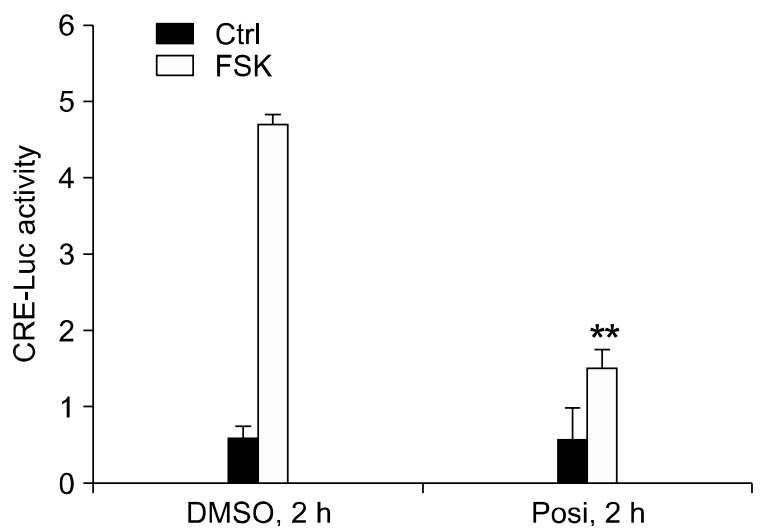

Figure 1. Adiponectin and rosiglitazone regulate gluconeogenic gene expression. ( $A$ and $B$ ) Effects of adiponectin on gluconeogenic gene expression (A) and CRE-luc activity (B) in primary hepatocytes $\left({ }^{*} P<0.05,{ }^{* *} P<0.01, n=3\right.$ each). Adenoviruses for adiponectin, AMPK CA and CRE-luc were infected for $16 \mathrm{~h}$ and then cultured for additional $24 \mathrm{~h}$ with $10 \mu \mathrm{M}$ forskolin (FSK) treatment for $2 \mathrm{~h}$ (for RT-PCR) or $4 \mathrm{~h}$ (for luciferase assay) before being harvested. (C and D) Effects of rosiglitazone on gluconeogenic gene expression $(C)\left({ }^{* *} P<0.01, n=3\right)$ and CRE-luc activity (D) $\left({ }^{* *} P<0.01, n=3\right.$ each) in primary hepatocytes. Adenoviruses for CRE-luc was infected for $16 \mathrm{~h}$ and then cultured for additional $48 \mathrm{~h}$ with $10 \mu \mathrm{M}$ forskolin (FSK) treatment for $2 \mathrm{~h}$ (for RT-PCR) or $4 \mathrm{~h}$ (for luciferase assay) before being harvested. In selected wells, $50 \mu \mathrm{M}$ rosiglitazone was treated for the final $2 \mathrm{~h}$ (for RT-PCR) or $4 \mathrm{~h}$ (for luciferase assay). Data in A-D represent mean \pm standard deviation from at least three independent experiments. 


\section{Effects of adiponectin and rosiglitazone require LKB1 expression}

LKB1 is an upstream kinase for activating AMPK and its related kinase families. Liver specific knockout of this gene in mice displayed impaired regulation of blood glucose levels and AMPK activation by metformin, suggesting it is an essential component for AMPK pathways in hepatocytes (Shaw et al., 2005). Having seen the effects of adiponectin on gluconeogenic gene expression, we wanted to confirm the importance of LKB1 in isolated primary hepatocyte setting. Indeed, knockdown of LKB1 via adenoviral shRNA blocks adiponectin-dependent inhibition of G6Pase and PGC-1 $\alpha$ expression as well as CRE luciferase activity (Figure 2A, 2B and 2D). Unexpectedly, rosiglitazone-mediated repression of gluconeogenic gene expression is also blunted by LKB1 knockdown in primary hepatocytes (Figure $2 \mathrm{C}$ ), suggesting that rosiglitazone-mediated pathway may also utilize LKB1-AMPK signaling cascades to modulate glucose metabolism.
A

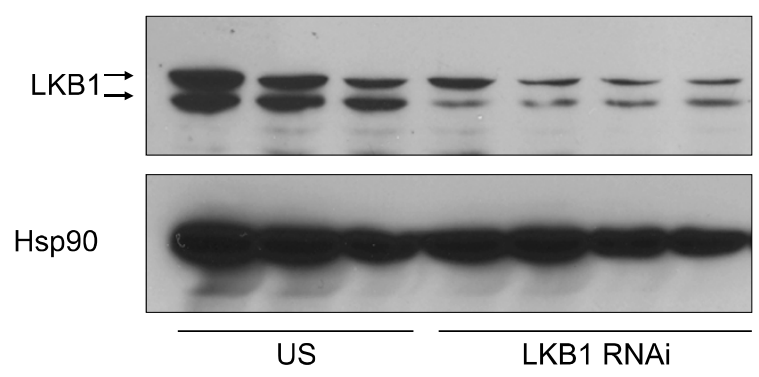

C
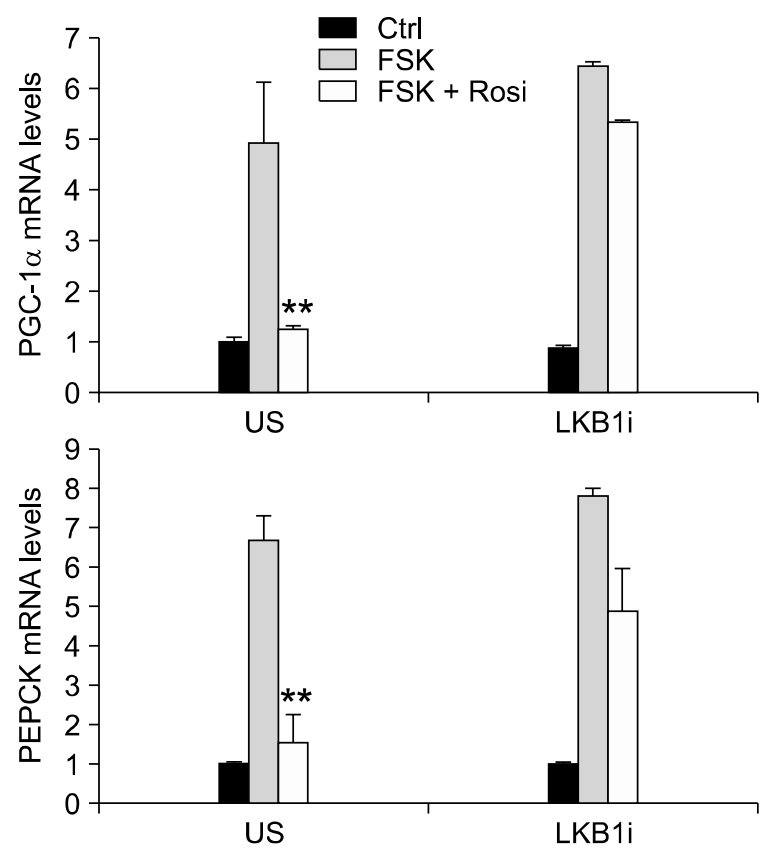

B

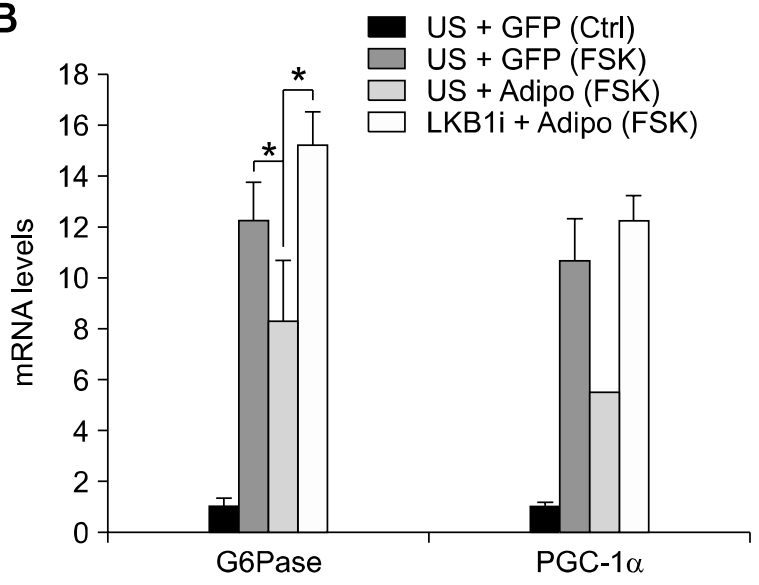

D

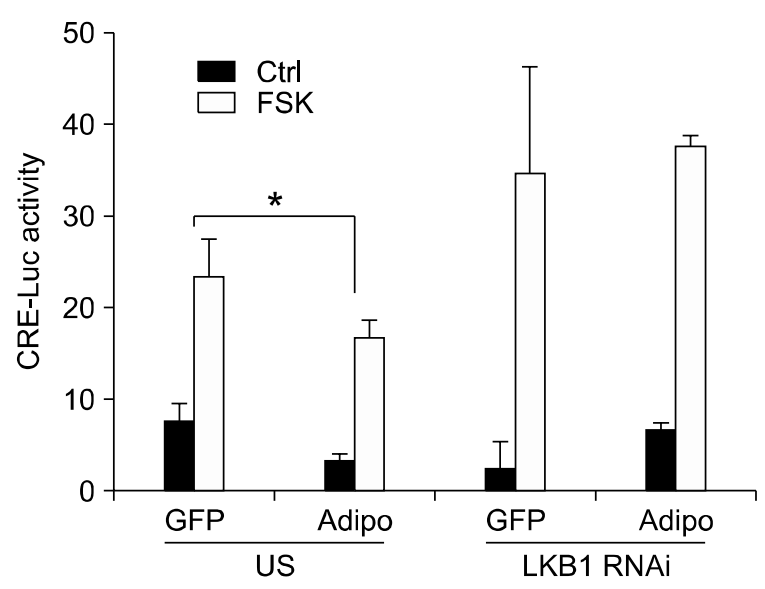

Figure 2. Effects of adiponectin and rosiglitazone require LKB1 expression. (A) Effects of LKB1 RNAi adenovirus on LKB1 expression in primary hepatocytes. Ad US (unspecific shRNA for control) or Ad LKB1 RNAi adenovirus was infected for $16 \mathrm{~h}$ and further maintained for additional $48 \mathrm{~h}$ in primary hepatocytes before being harvested for western blot analysis. (B and C) Effects of LKB1 knockdown on adiponectin-dependent (B) or rosiglitazone-dependent $(C)$ regulation on gluconeogenic gene expression in primary hepatocytes $\left({ }^{*} P<0.05,{ }^{* *} P<0.01, n=3\right.$ each). (D) Effects of LKB1 knockdown on adiponectin-dependent regulation on CRE-luc activity in primary hepatocytes ( ${ }^{*} P<0.05 n=3$ each) Adenoviruses for adiponectin and LKB1 RNAi were infected for $16 \mathrm{~h}$ and then cultured for additional $48 \mathrm{~h}$ with $10 \mu \mathrm{M}$ forskolin (FSK) treatment for $2 \mathrm{~h}$ (for RT-PCR) or $4 \mathrm{~h}$ (for luciferase assay) before being harvested. In selected wells, $50 \mu \mathrm{M}$ rosiglitazone was treated for the final $2 \mathrm{~h}$. Data in B-D represent mean \pm standard deviation from at least three independent experiments. 


\section{Adiponectin and rosiglitazone are direct inducers for AMPK and promote CRTC2 phosphorylation}

Adiponectin is shown to activate AMPK pathway in liver. Indeed, adenovirus-mediated expression of adiponectin induces phosphorylation of Thr 172 of AMPK in primary hepatocytes (Figure $3 \mathrm{~A}$ ). As suggested by the lack of effects of rosiglitazone on gluconeogenic gene expression with LKB1 knockdown (Figure 2C), treatment of rosiglitazone also stimulate phosphorylation of AMPK in hepatocytes (Figure 3B). Indeed, activation of AMPK enhances phosphorylation of CRTC2, as evidenced by the emergence of slower migrating bands in western

A

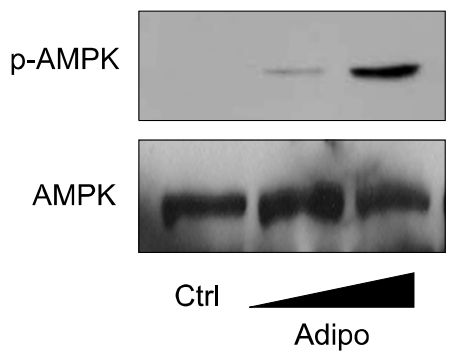

C
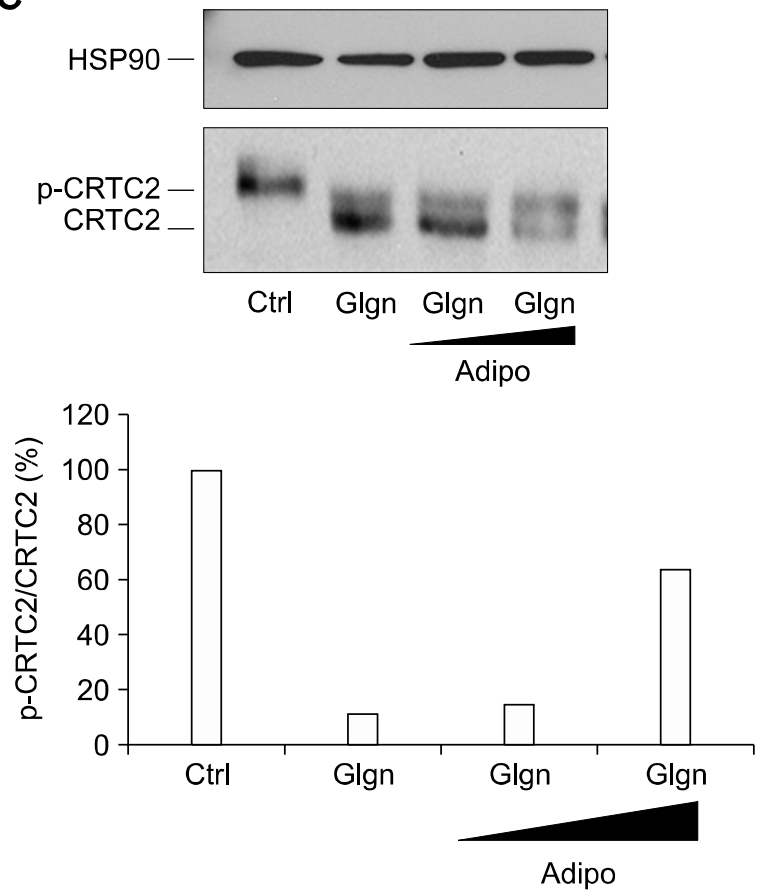

blot analysis for both adiponectin (Figure 3C) and rosiglitazone (Figure 3D) treated samples. Moreover, the effects of adiponectin (Figure 4A) or rosiglitazone (data not shown) on CRE activity is effectively blocked by co-expression of phosphorylation defective mutant CRTC2 (S171A CRTC2), but not by wild type CRTC2, suggesting that modulation of gluconeogenic gene expression by both reagents include CRTC2 phosphorylation events in liver. Collectively, these data support the hypothesis that not only adiponectin but also rosiglitazone could directly regulate hepatic gluconeogenesis via AMPK-mediated regulation of CRTC2.

B

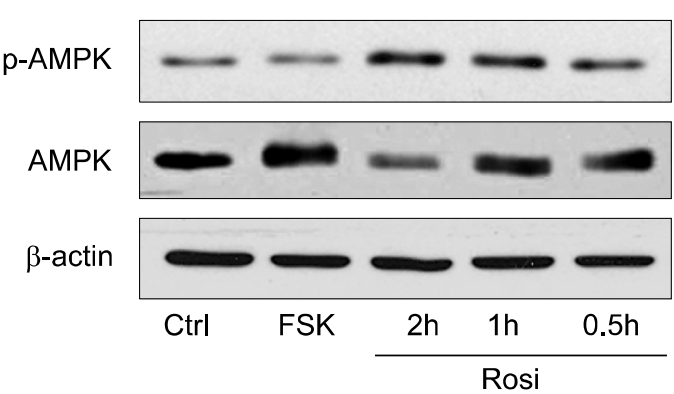

D
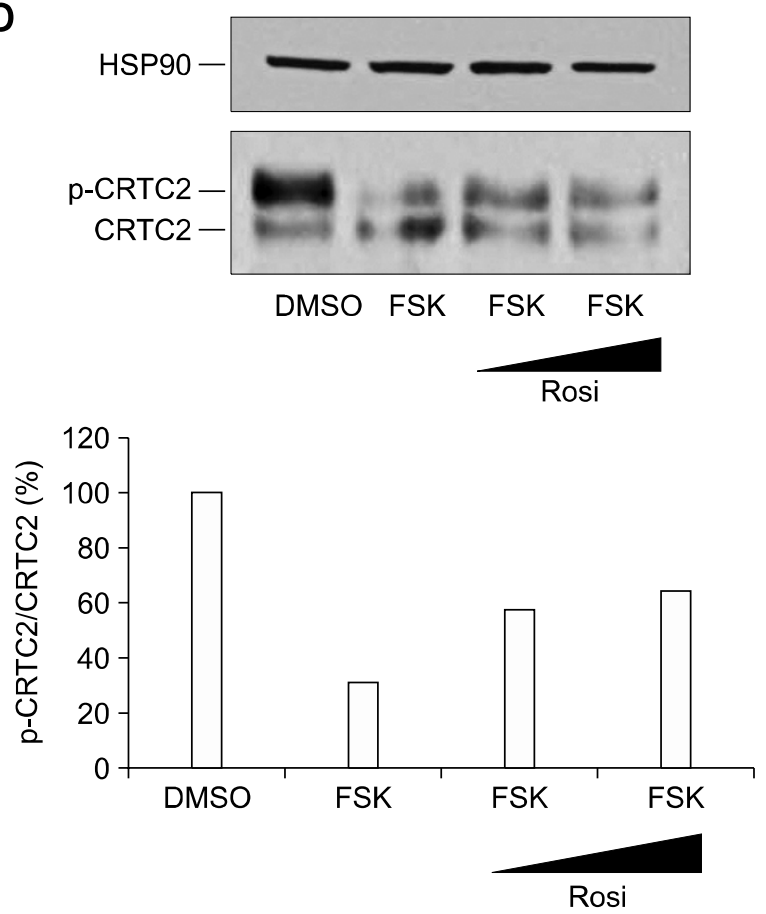

Figure 3. Adiponectin and rosiglitazone regulate CRTC2 phosphorylation. (A and B) Effects of adiponectin (A) and rosiglitazone (B) on AMPK phosphorylation in primary hepatocytes. (C and D) Effects of adiponectin (A) and rosiglitazone (B) on CRTC2 phosphorylation in primary hepatocytes. For experiments in ( $\mathrm{A}$ and $\mathrm{C}$ ) adenovirus for adiponectin was infected for $16 \mathrm{~h}$ and then cultured for additional $24 \mathrm{~h}$ prior to $100 \mathrm{nM}$ glucagons (Glgn) treatment for 10 min before being harvested. For experiments in (B), $50 \mu \mathrm{M}$ rosiglitazone was treated for various time points indicated with 10 min-treatment of $10 \mu \mathrm{M}$ forskolin. For experiments in (D), $50 \mu \mathrm{M}$ or $100 \mu \mathrm{M}$ rosiglitazone was treated for $2 \mathrm{~h}$ with 10 min-treatment of $10 \mu \mathrm{M}$ forskolin. The data represent three independent experiments with similar results. 
A

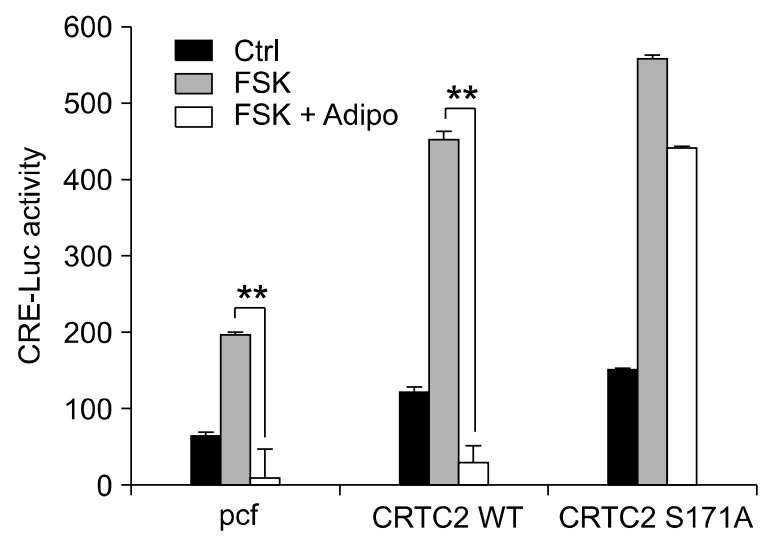

C

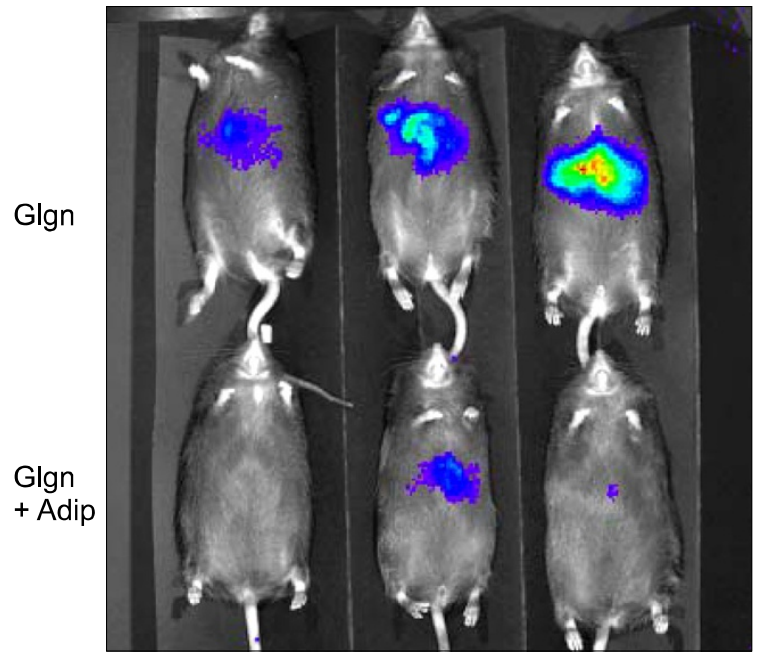

B
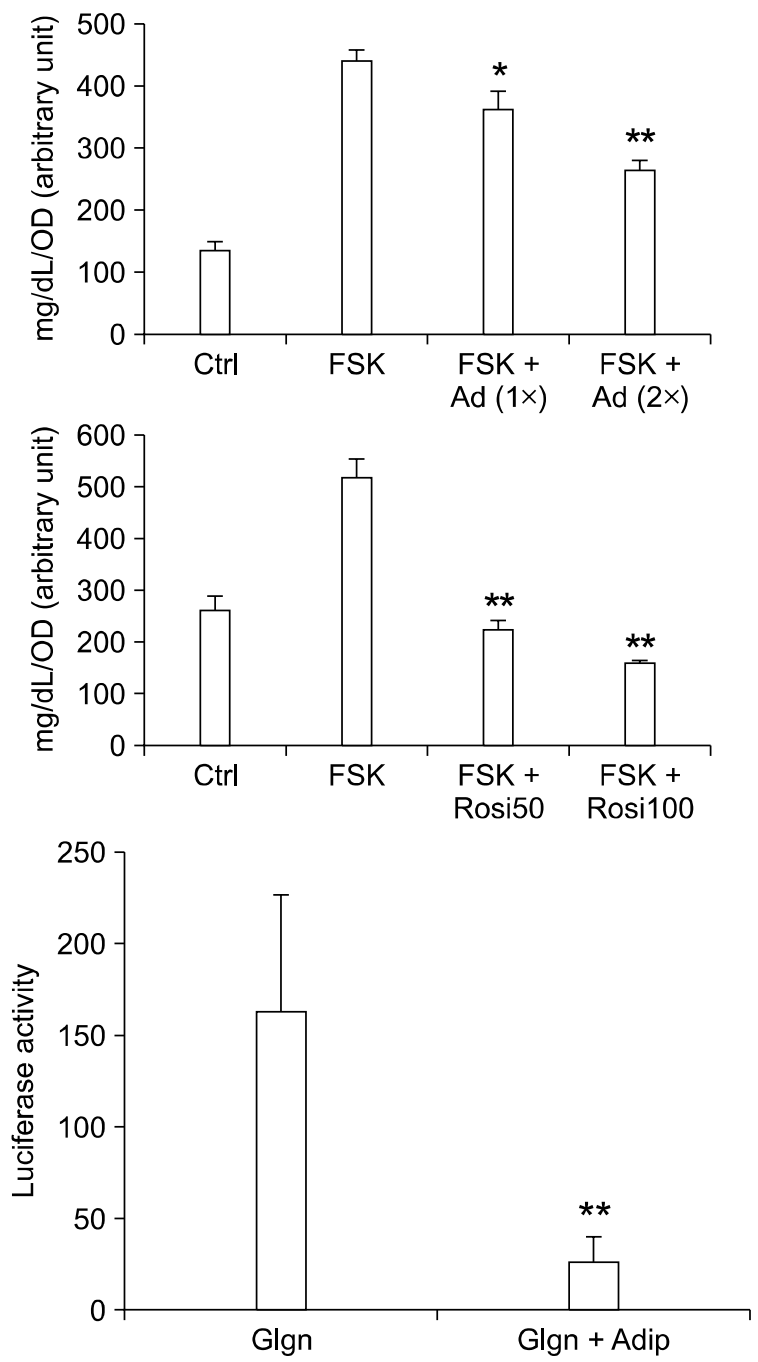

Figure 4. Adiponectin and rosiglitazone regulate hepatic glucose production. (A) Effects of adiponectin on CRTC2-dependent regulation of CRE activity in primary hepatocytes ( ${ }^{* *} P<0.01, n=3$ each). Adenoviruses for adiponectin, WT or S171A CRTC2 and CRE-luc were infected for $16 \mathrm{~h}$ and then cultured for additional $24 \mathrm{~h}$ with $10 \mu \mathrm{M}$ forskolin (FSK) treatment for $4 \mathrm{~h}$ (for luciferase assay) before being harvested. (B) Effects of adiponectin (top) and rosiglitazone (bottom) on glucose production in primary hepatocytes ( ${ }^{*} P<0.05,{ }^{*} P<0.01, n=3$ each). Glucose output assay was performed using primary hepatocytes with $16 \mathrm{~h}$-infection of Ad adiponectin or $12 \mathrm{~h}$-treatment of 50-100 $\mu \mathrm{M}$ rosiglitazone in the absence or in the presence of $10 \mu \mathrm{M}$ forskolin (FSK). (C) Effects of Ad-adiponectin on hepatic Ad-CRE luc activity. In vivo luminescence was measured 5 days post adenovirus infection as described in methods. Graphic representation of ROI (left) and its quantitation (right) shown ( ${ }^{* *} P<0.01, n=3$ each). Data in A-C represent mean \pm standard deviation from at least three independent experiments.

\section{Adiponectin and rosiglitazone regulate hepatic glucose production}

Next, we wanted to confirm whether changes in hepatic gluconeogenic gene expression by adiponectin or rosiglitazone would lead to the alteration in glucose production. We thus performed glucose output assay in primary hepatocytes treated with either rosiglitazone or adiponectin to monitor the changes in glucose production. Treatment of forskolin greatly enhances glucose production up to 2to 3-fold compared with DMSO control (Figure 4B).
The effects of forskolin-dependent increase in hepatic glucose production are largely blunted by either adiponectin or rosiglitazone treatment, showing indeed both reagents could directly reduce glucose output from hepatocytes. Finally, we wanted to confirm whether the ability of adiponectin to regulate CRE-mediated transcription in isolated hepatocytes could be recapitulated in vivo. We thus turn to utilize in vivo imaging system to monitor the dynamic changes in hepatic CRE activity in vivo in a non-invasive manner. As in the 
case of regulation of CRE luciferase in primary hepatocytes, injection of glucagon greatly enhances CRE luciferase activity in livers of mice infected with Ad-GFP control viruses (Figure 4C). On the other hand, mice infected with Ad-adiponectin adenoviruses show close to 80 percent reduction in CRE luciferase activity compared with mice with control viruses, showing indeed adiponectin could inhibit cAMP-mediated transcription in mouse liver.

\section{CRTC2 is a focal point for the regulation of hepatic glucose production}

Following its identification as a CREB coactivator, CRTC2 has been demonstrated as a critical transcriptional regulator for the hepatic gluconeogenesis. Although various post-translational modifications such as O-glycosylation and acetylation/deacetylation were shown to be critical for the regulation of CRTC2 activity (Dentin et al., 2008, Liu et al., 2008 ), the primary modulation of this factor is achieved by serine 171 phosphorylation via AMPK family of serine/threonine kinases (Screaton et al., 2004; Koo et al., 2005). The regulation of CRTC2 phosphorylation by adiponectin-mediated induction of AMPK was expected in part due to the studies showing the direct role of adiponectin in activating hepatic AMPK signaling pathway. However, the direct involvement of rosiglitazone in regulating hepatic CRTC2 activity via LKB1-AMPK pathway would provide a novel molecular mechanism by which this reagent would modulate plasma glucose levels. Collectively, these data underscore the importance of CRTC2 in the regulation of hepatic glucose production. Development of specific inhibitors for CRTC2 activity would provide an improved means for modulating hyperglycemia for individuals with type II diabetes.

\section{Methods}

\section{Culture of primary hepatocytes}

Rat primary hepatocytes were prepared from SpragueDawley rats as described previously (Koo et al., 2005). After attachment, cells were infected with adenoviruses for $16 \mathrm{~h}$, and were further maintained in medium 199 (MediaTech) without serum for an additional $24 \mathrm{~h}$. Cells were treated with $10 \mu \mathrm{M}$ forskolin or $100 \mathrm{nM}$ glucagon for the final $10 \mathrm{~min}$ (for western blot), $2 \mathrm{~h}$ (for RT PCR) or $4 \mathrm{~h}$ (for luciferase assay). In experiments indicated in the legend, 50 to $100 \mu \mathrm{M}$ rosiglitazone was treated for $4-8 \mathrm{~h}$.

\section{Adenoviruses}

Ad-US (unspecific shRNA), Ad-GFP and Ad-CRE-luc was described previously (Herzig et al., 2003; Dentin et al.,
2007). cDNA for adiponectin was amplified from mouse adipose tissues. Mouse liver cDNA was used to amplify sequences for AMPK $\alpha 2$ (aa 1-342). A point mutation bearing sequences encoding Asp instead of Thr at amino acid 172 was generated by QuikChange Site directed mutagenesis kit (Stratagene) as described previously (Lee and Allen, 2007). Adenoviruses for LKB1 RNAi, adiponectin and AMPK $\alpha 2$ T172D 1-342 (AMPK CA) were generated as described (Koo et al., 2005).

\section{Glucose output assay}

Glucose output from primary hepatocytes was determined enzymatically as described previously (Dentin et al., 2007).

\section{Animal experiments}

Male, 7-week-old C57BL/6 mice were purchased from Charles River Laboratories. Recombinant adenovirus (0.5 $\times 10^{9}$ PFU) was delivered by tail-vein injection to mice. For imaging, $18 \mathrm{~h}$-fasted mice with Ad-CRE-luc and Ad-GFP or Ad-CRE-luc and Ad-adiponectin were injected intraperitoneally with $100 \mathrm{mg} / \mathrm{kg}$ sterile D-luciferin. Mice were then anaesthetized, and imaged on the IVIS 200 Imaging System (Xenogen) as described previously (Dentin et al., 2007).

\section{Western blot analysis}

Western blot analysis using $50-100 \mu \mathrm{g}$ of protein from whole cell lysates were performed as described previously (Koo et al., 2004). Polyclonal antibody against CRTC2 was used as described previously (Screaton et al., 2004). Antibodies against LKB1, AMPK and p-AMPK were purchased from cell signaling. Antibodies against HSP90 (Santa Cruz) was used to assess equal loading.

\section{Quantitative PCR}

Total RNA from primary hepatocytes was extracted using RNeasy mini-kit (Qiagen). cDNAs generated by Superscript II enzyme (Invitrogen) were analyzed by quantitative PCR (Q-PCR) using a SYBR green PCR kit and TP800 Thermal Cycler DICE Real Time System (TAKARA). All data were normalized to ribosomal L32 expression.

\section{Statistical analyses}

Results represent as mean \pm standard deviation. The comparison of different groups was carried out using two-tailed unpaired Student's $t$-test as described (Dentin et al., 2007). Differences were considered statistically significant at $P<0.05$.

\section{Acknowlegements}

We would like to thank Professor Marc Montminy for the critical comments for our work. We would also like to thank Sun Myung Park for the technical assistance. This work was supported by the Research Program for New Drug Target Discovery (M10648000089-08N4800-08910) grant, 
Korea Science and Engineering Foundation (KOSEF) grant (R01-2008-000-11935-0), and Korea Research Foundation (KRF) Grant (2006-E00037) by the Ministry of Education, Science and Technology, Republic of Korea.

\section{References}

Arias J, Alberts AS, Brindle P, Claret FX, Smeal T, Karin M, Feramisco J, Montminy M. Activation of cAMP and mitogen responsive genes relies on a common nuclear factor. Nature 1994;370:226-9

Chrivia JC, Kwok RP, Lamb N, Hagiwar M, Montminy MR, Goodman RH. Phosphorylated CREB binds specifically to the nuclear protein CBP. Nature 1993;365:855-9

Dentin R, Liu Y, Koo SH, Hedrick S, Vargas T, Heredia J, Yates $\mathrm{J}$ 3rd, Montminy M. Insulin modulates gluconeogenesis by inhibition of the coactivator TORC2. Nature 2007;449: 366-9

Dentin R, Hedrick S, Xie J, Yates J 3rd, Montminy M. Hepatic glucose sensing via the CREB coactivator CRTC2. Science 2008;319:1402-5

Foretz M, Ancellin N, Andreelli F, Saintillan Y, Grondin P, Kahn A, Thorens B, Vaulont S, Viollet B. Short-term overexpression of a constitutively active form of AMP-activated protein kinase in the liver leads to mild hypoglycemia and fatty liver. Diabetes 2005;54:1331-9

Hall RK, Granner DK. Insulin regulates expression of metabolic genes through divergent signaling pathways. J Basic Clin Physiol Pharmacol 1999;10:119-33

Hanson RW, Reshef L. Regulation of phosphoenolpyruvate carboxykinase (GTP) gene expression. Annu Rev Biochem 1997;66:581-611

He W, Barak Y, Hevener A, Olson P, Liao D, Le J, Nelson M, Ong E, Olefsky JM, Evans RM. Adipose-specific peroxisome proliferator-activated receptor gamma knockout causes insulin resistance in fat and liver but not in muscle. Proc Natl Acad Sci U S A 2003;100:15712-7

Herzig S, Long F, Jhala US, Hedrick S, Quinn R, Bauer A, Rudolph D, Schutz G, Yoon C, Puigserver P, Spiegelman B, Montminy $M$. CREB regulates hepatic gluconeogenesis through the coactivator PGC-1. Nature 2001;413:179-83

Herzig S, Hedrick S, Morantte I, Koo SH, Galimi F, Montminy M. CREB controls hepatic lipid metabolism through nuclear hormone receptor PPAR-gamma. Nature 2003;426:190-3

Koo SH, Satoh H, Herzig S, Lee CH, Hedrick S, Kulkarni R, Evans RM, Olefsky J, Montminy M. PGC-1 promotes insulin resistance in liver through PPAR-alpha-dependent induction of TRB-3. Nat Med 2004;10:530-4

Koo SH, Flechner L, Qi L, Zhang X, Screaton RA, Jeffries S., Hedrick S, Xu W, Boussouar F, Brindle P, Takemori H, Montminy $M$. The CREB coactivator TORC2 is a key regulator of fasting glucose metabolism. Nature 2005;437: 1109-11

Lebrasseur NK, Kelly M, Tsao TS, Farmer SR, Saha AK, Ruderman NB, Tomas E. Thiazolidinediones can rapidly activate AMP-activated protein kinase in mammalian tissues. Am J Physiol Endocrinol Metab 2006;291:E175-81

Lee EH, Allen PD. Homo-dimerization of RyR1 C-terminus via charged residues in random coils or in an alpha-helix. Exp Mol Med 2007;39:594-602

Liu Y, Dentin R, Chen D, Hedrick S, Ravnskjaer K, Schenk S, Milne J, Meyers DJ, Cole P, Yates J 3rd, Olefsky J, Guarente L, Montminy M. A fasting inducible switch modulates gluconeogenesis via activator/coactivator exchange. Nature 2008;456:269-73

Nawrocki AR, Rajala MW, Tomas E, Pajvani UB, Saha AK, Trumbauer ME, Pang Z, Chen AS, Ruderman NB, Chen H, Rossetti L, Scherer PE. Mice lacking adiponectin show decreased hepatic insulin sensitivity and reduced responsiveness to peroxisome proliferator-activated receptor gamma agonists. J Biol Chem 2006;281:2654-60

Screaton RA, Conkright MD, Katoh Y, Best JL, Canettieri G, Jeffries S, Guzman E, Niessen S, Yates JR 3rd, Takemori H, Okamoto M, Montminy M. The CREB coactivator TORC2 functions as a calcium- and cAMP-sensitive coincidence detector. Cell 2004;119:61-74

Shaw RJ, Lamia KA, Vasquez D, Koo SH, Bardeesy N, Depinho RA, Montminy M, Cantley LC. The kinase LKB1 mediates glucose homeostasis in liver and therapeutic effects of metformin. Science 2005;310:1642-6

Zhou G, Myers R, Li Y, Chen Y, Shen X, Fenyk-melody J, Wu M, Ventre J, Doebber T, Fujii N, Musi N, Hirshman MF, Goodyear LJ, Moller DE. Role of AMP-activated protein kinase in mechanism of metformin action. J Clin Invest 2001;108:1167-74 